\title{
RADIOPROTECTION CALCULATIONS FOR MEGAPIE
}

\author{
L. Zanini* \\ Paul Scherrer Institut, CH-5232 Villigen PSI, Switzerland
}

\begin{abstract}
The MEGAwatt PIlot Experiment (MEGAPIE) liquid lead-bismuth spallation neutron source will commence operation in 2006 at the SINQ facility of the Paul Scherrer Institut. Such an innovative system presents radioprotection concerns peculiar to a liquid spallation target. Several radioprotection issues have been addressed and studied by means of the Monte Carlo transport code, FLUKA. The dose rates in the room above the target, where personnel access may be needed at times, from the activated lead-bismuth and from the volatile species produced were calculated. Results indicate that the dose rate level is of the order of $40 \mathrm{mSv} \mathrm{h}^{-1} 2 \mathrm{~h}$ after shutdown, but it can be reduced below the $\mathrm{mSv} \mathrm{h}{ }^{-1}$ level with slight modifications to the shielding. Neutron spectra and dose rates from neutron transport, of interest for possible damage to radiation sensitive components, have also been calculated.
\end{abstract}

\section{INTRODUCTION}

The MEGAwatt PIlot Experiment (MEGAPIE) project $^{(1)}$ was started in 2000 to design, build and operate a liquid metal spallation neutron target of 1 MW beam power. The project is an important step in the roadmap towards the demonstration of the ADS (accelerator driven system) concept ${ }^{(2)}$ and high-power molten metal targets in general. The target will be installed at the SINQ facility at the Paul Scherrer Institut, and irradiated for one year in 2006. The target will be inserted into the existing SINQ facility, replacing the solid 'cannelloni target $^{(3)}$ installed at present.

The target design strongly depends on the support provided by the research groups investigating the neutronics, thermo-hydraulic, materials, safety and reliability issues. From the neutronic and nuclear point of view, many important aspects were addressed in the design phase of MEGAPIE, such as the calculation of the neutron flux, the beam power deposition, the radionuclide inventory and the radiation damage to structural materials and other components ${ }^{(4-6)}$.

The SINQ facility is properly shielded as in the design phase the different sources of radiation typical of a spallation source were considered $^{(7,8)}$. On the other hand, a liquid metal target such as MEGAPIE introduces new radioprotection issues related to the lead-bismuth eutectic (LBE) loop. One of the key points of the design concerns the top part of the target, which is rather complex but must be designed keeping in mind the need to minimise the effective dose rates due to the irradiation and activation of the target. It is important to determine the effective dose rates due to radiation coming from the target since this room [so-called TKE (target head enclosure)] may need to be accessed at times for maintenance operations, a few hours after

${ }^{*}$ Corresponding author: Luca.Zanini@psi.ch beam shutdown. For this purpose, one must consider radiation coming from the target activation. The LBE in MEGAPIE circulates in a loop that extends vertically for $3 \mathrm{~m}$ along most of the length of the target; it will be activated during operation, and at the surface level, at the top of the target, the gamma source strength may be high enough to be of concern for personnel access. Moreover, part of the activated LBE will be in the form of radioactive gas and volatile elements; these will accumulate in an expansion tank placed above the LBE surface, inside the target at a level near the TKE floor, and the radiation level from this component may also be of concern. The different components of the radiation coming from the target were considered and the shielding was designed based on these indications ${ }^{(9)}$. However, given the complexity of the MEGAPIE target design, which must include oil and heavy-water loops in the top part, possible weak points in the top part of the shielding have been indicated. It is therefore important to perform a gamma radiation transport study, in which the geometry of the target must be detailed in the best possible manner and include the existing gaps in the shielding.

Another source of concern is the radiation coming from the spallation neutrons streaming from the bottom of the target to the top. This component is present only during irradiation and it is not a concern for the access to the TKE. However, it is important to determine the flux of neutrons in the TKE since a high neutron flux can damage components of the MEGAPIE target and its ancillary systems. This component of the radiation will add to the neutron and gamma radiation from the heavy-water cooling circuits, which was studied previously ${ }^{(9)}$.

In this work, radioprotection calculations related to radiation coming from the target are presented. The FLUKA code ${ }^{(10)}$, version 2003.1, was used. The main focus was the evaluation of the effective dose rate in the TKE from the target, with and without 


\section{ZANINI}

the beam. The following sources of radiation have been considered in the calculations: (1) gamma radiation from the top part of the LBE loop; (2) gamma radiation from the cover gas plenum. The transport of spallation neutrons from the target bottom up to the TKE is also calculated.

\section{CALCULATION PROCEDURE}

\section{Activation calculations}

A model of the MEGAPIE target was developed using the FLUKA code. An attempt was made to reproduce the actual target design ${ }^{(11)}$, in a satisfactory manner for the purposes of the present investigation. The FLUKA model of the MEGAPIE target and the surrounding SINQ facility is shown in Figure 1. The structure of the top part of the target is very complex since in this zone of the target there are several components such as the gas expansion tank and the water and oil distribution boxes. It is therefore a region where there is a lower amount of stainless steel and several gaps are present. A layer of tungsten was added in this region for shielding purposes. An attempt was made to reproduce this part in some detail, however, approximations must be made, and whenever it was not possible to detail the geometry, stainless steel of lower density $(65 \%$ of the nominal density) was used to account for small gaps.

The following procedure was applied in the calculations: first, the radionuclide inventory of the $\mathrm{LBE}$, for different irradiation times at $1.4 \mathrm{~mA}$, was calculated. Calculations of MEGAPIE irradiation were performed using a source proton beam of $575 \mathrm{MeV}$ energy as input, with the spatial profile experimentally measured at $\mathrm{SINQ}^{(12)}$. In the procedure for the gas handling, it is foreseen that the gas produced will be collected for up to $30 \mathrm{~d}$ in the

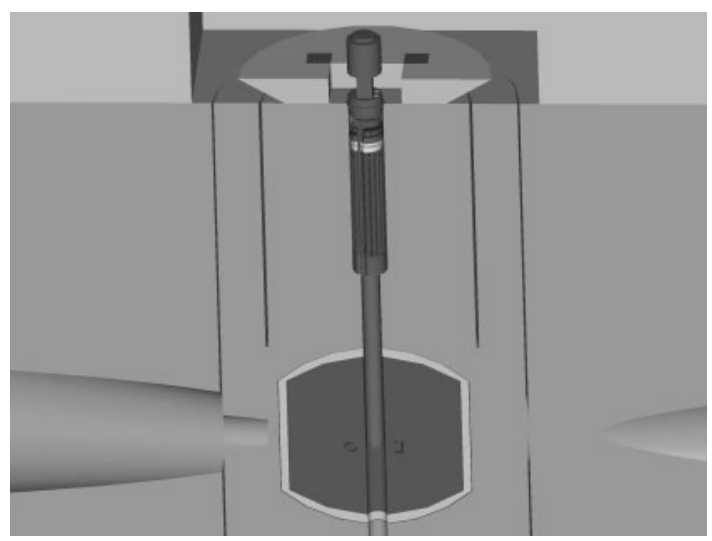

Figure 1. FLUKA geometry of the MEGAPIE target and the surrounding SINQ facility. expansion tank placed at the target top. After this period the gas will be transferred to a decay tank placed in the TKE, where it will be left to decay for an additional $30 \mathrm{~d}$, before venting it to the exhaust system.

To calculate the activities, the output from FLUKA consisting of a list of the production rates (atoms/proton) for all the isotopes was used. The production rates were then used by the ORIHET3 program $^{(13)}$ which performs the calculation of the activity build-up and decay at times specified by the user. In the present work, delay times of the order of hours were considered, while for other studies, for instance studies related to the target disposal, longer decay times can be considered. ORIHET3 calculates the mass, the radioactivity of unstable isotopes and the intensities of the emitted gamma lines for each isotope produced. By taking into account the radioisotopes of interest (all isotopes or only the volatile elements) it is possible to calculate the gamma source strength as a function of the energy.

It is important to separate the contributions of the volatile elements from the total since the volatile elements will be collected in the target expansion tank, which is placed at a higher position with respect to the LBE surface level and is potentially more dangerous. There may be a contribution from the activation of the structural materials also, but it is negligible compared with the LBE in which most of the beam energy is deposited. Besides the noble gases, most of the activity of the elements with high volatility comes from mercury. In order to distinguish the contribution from the volatile elements, one has to make a somewhat arbitrary separation, as in some cases there is uncertainty whether an element is volatile or not.

After the gamma source strength is obtained, the FLUKA is run once again but this time the photon transport is applied according to the appropriate source strength. The source is placed in the top part of the LBE loop or inside the expansion tank. A map of the photon flux and of the dose rates can be obtained with the proper normalisation to the gamma source strength.

\section{RESULTS}

\section{Activation}

The activity build-up in the LBE for $200 \mathrm{~d}$ and subsequent decay was calculated. The total activity of $\sim 2 \times 10^{16} \mathrm{~Bq}$ is reached after a few days; after shutdown the activity drops by more than one order of magnitude in 1 month. The gamma-emitting radionuclides give a total gamma source strength which has a similar behaviour, as shown in Figure 2. The total gamma source strength is evenly distributed in the 801 of the LBE. The contribution of the noble 


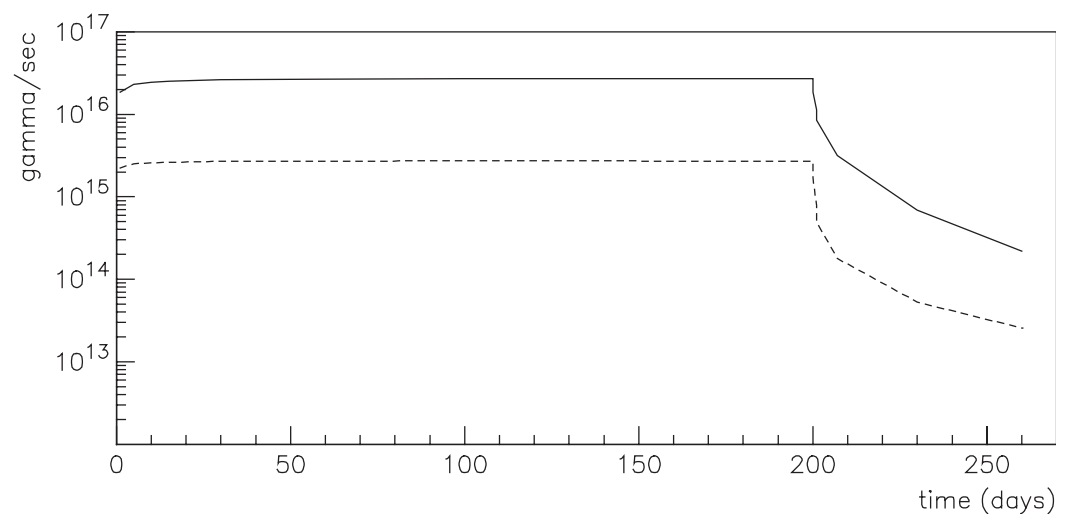

Figure 2. Total gamma source strengths for all elements (full lines) and for the volatile elements (dashed lines) in the LBE at different build-up and decay times. Proton beam current $=1.4 \mathrm{~mA}$.

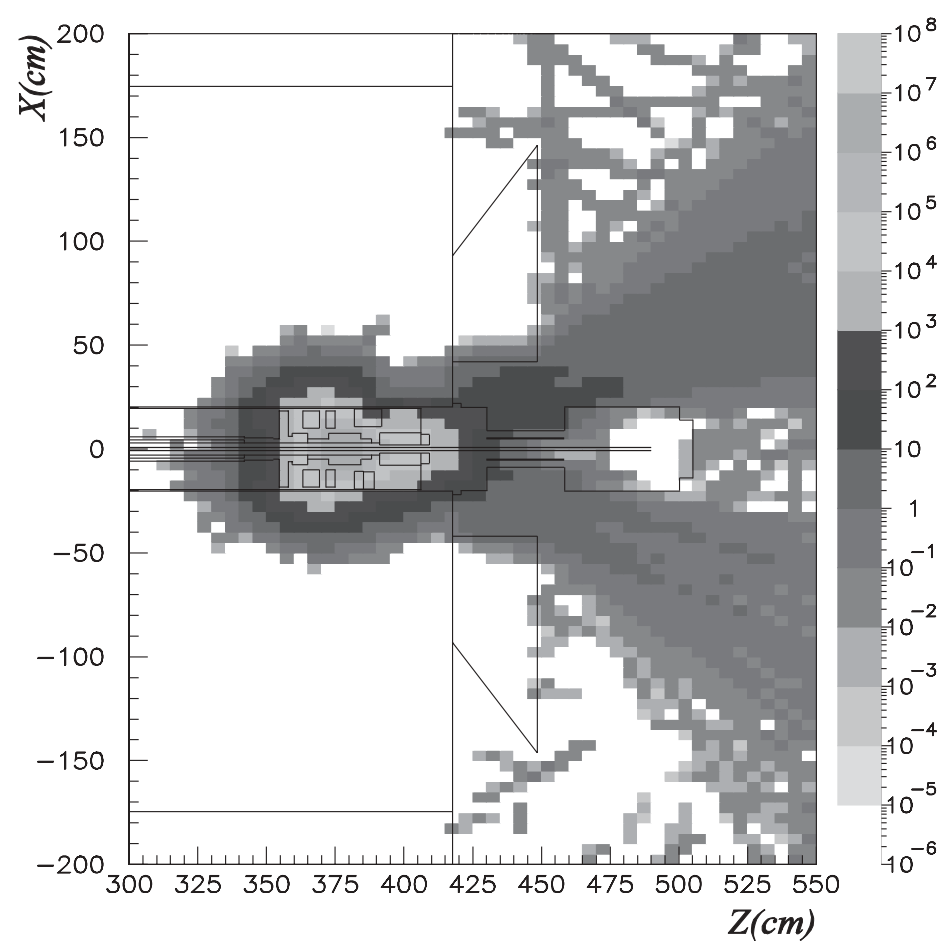

Figure 3. Effective dose rate in $\mathrm{mSv} \mathrm{h}^{-1}$ in the top part of MEGAPIE and in the TKE room due to the gamma radiation from activated gas in the target expansion tank, one week after shutdown.

gases plus the elements with high volatility is about one order of magnitude lower than the total activity. Most of this component will collect in the expansion tank. The gamma spectra for volatile elements and all nuclei at different cooling times are obtained from ORIHET3. It is important to mention that at longer cooling times the spectra become softer and, therefore, one must consider the proper gamma spectra in the dose rate calculations.

\section{Dose rates in TKE}

From the source strength distributions, photon transport calculations are performed by placing the sources at the LBE surface and in the expansion tank.

In Figure 3 an $X Z$ map of the effective dose rates in $\mathrm{mSv} \mathrm{h}^{-1}$ due to gamma radiation coming from the target expansion tank is shown. The figure refers to 


\section{ZANINI}

Table 1. Effective dose rates $\left(\mathrm{mSv} \mathrm{h}^{-1}\right)$ in the TKE floor following shutdown.

\begin{tabular}{lccrcc}
\hline & $t_{0}$ & $2 \mathrm{~h}$ & $12 \mathrm{~h}$ & $1 \mathrm{~d}$ & 1 week \\
\hline ET & 70 & 40 & 30 & 22 & 10 \\
LBE & 3.5 & 1.2 & 1 & 0.5 & 0.2 \\
\hline
\end{tabular}

Contribution from the expansion tank (ET) and the LBE surface are indicated

the dose rates one week after shutdown. As shown, the shielding is not sufficient to prevent a significant amount of radiation from entering the TKE. The highest dose rate $2 \mathrm{~h}$ after shutdown (at the floor near the target head) is $40 \mathrm{mSv} \mathrm{h}$. The indicative values extracted from these two-dimensional plots at the level of the TKE floor are shown in Table 1. The same calculation was performed by adding a layer of $15 \mathrm{~cm}$ of lead around the target head to cover the hole in the shielding between $425<z<450$. Since this gap can only be filled with lead powder, a density of $60 \%$ of the nominal lead density was assumed. In this case most of the radiation is stopped to a level well below the acceptable limits $\left(<1 \mathrm{mSv} \mathrm{h}^{-1}\right)$.

For the radiation from the LBE surface, the dose rate level is much lower, since the LBE is placed below the cover gas plenum and the existing stainless steel layer in conjunction with the tungsten layers provides sufficient shielding.

\section{Neutrons}

The integrated neutron flux in the TKE is $\sim 3 \times$ $10^{6} \mathrm{n} \mathrm{cm}^{-2} \mathrm{~s}^{-1}$, with a component of nearly $2 \times$ $10^{5} \mathrm{n} \mathrm{cm}^{-2} \mathrm{~s}^{-1}$ of neutrons with $E>1 \mathrm{MeV}$. It is important to note that this flux refers only to neutrons coming from the target region and that it does not take into account the contribution from neutrons scattered from the TKE walls. According to Ref. (7) this contribution can be of the same order of magnitude, even though the spectrum will be softer. A strong neutron flux also originates from the decay of light elements from the activation of the heavywater loop ${ }^{(9,14)}$. Such a radiation field can be of concern for radiation-sensitive components to be used in the MEGAPIE, some of which use organic components. The manufacturers of these components have been requested to ensure that the components qualify for these radiation levels.

\section{CONCLUSIONS}

The Monte Carlo FLUKA code in conjunction with the ORIHET3 code was applied in the study of radiation protection aspects related to the liquid lead-bismuth spallation target MEGAPIE. Emphasis was given to the effective dose rates at the top of the target originating from activation of the LBE inside the target.

The greatest contribution to the effective dose rate after shutdown comes from activated gases and volatile elements which accumulate in the target expansion tank. To effectively shield this radiation an additional layer of $\sim 15 \mathrm{~cm}$ of lead is necessary.

The neutron flux in the TKE was also calculated. The flux of neutrons from the target is at the level of $3 \times 10^{6} \mathrm{n} \mathrm{cm}^{-2} \mathrm{~s}^{-1}$, with a relevant fraction of fast and high-energy neutrons. Radiation-sensitive components must be qualified to sustain these flux levels, including the contribution from the activated heavy-water loop. Future calculations will include activation calculations in the TKE induced by the neutron flux.

\section{REFERENCES}

1. Bauer, G. S., Salvatores, M. and Heusener, G. MEGAPIE, a $1 \mathrm{MW}$ pilot experiment for a liquid metal spallation target. J. Nucl. Mat. 296, 17 (2001).

2. The European technical working group on ADS. A European roadmap for developing ADS for nuclear waste incineration (2001).

3. Bauer, G. S. Spallation neutron source development at the Paul Scherrer Institut. In: Proceedings of the Embedded Topical Meeting on Accelerator Applications, Albuquerque, NM 16-20 November (1997).

4. Pitcher, E. Private communication (2002).

5. Foucher, Y. Etude et développement d'une cible de spallation, $\mathrm{PhD}$ thesis, Université de Nantes (2002).

6. Pitcher, E. Radiation dose received by diphyl in the MEGAPIE primary heat exchanger. PSI report AN-34-02-04 (2002).

7. Atchison, F. Dose-rates in TKE with 'real' target. PSI internal report 816/AF30-306 (1993).

8. Atchison, F. Neutronic considerations in the design of SINQ. PSI report SINQ/816/AFN-703 (1987).

9. MEGAPIE Safety Report (2002).

10. Fasso, A., Ferrari, A., Ranft, J. and Sala, P. R. FLUKA: status and prospective for hadronic applications. In: Proceedings of the MonteCarlo 2000 Conference, Lisbon, 23-26 October 2000, Kling, A., Barao, F., Nakagawa, M., Tavora, L. and Vaz, P. Eds. (Berlin: Springer-Verlag), pp. 955-960 (2001)

11. Kirchner, T. et al. MEGAPIE target design and dimensioning. In: Proceedings of the fourth MEGAPIE Technical Review Meeting, Fazio, C., Knebel, J. U. and Gröschel, F. Eds. Paris, France, 18-19, March (2003).

12. Rohrer, U. Private communication (2001).

13. Atchison, F. and Schaal, H. Orihet 3-Version 1.12. A guide for users (2001).

14. Geissmann, K. Private communication (2002). 